\title{
Smart battles: immunosuppression versus immunomodulation in the inflammatory RMDs
}

\author{
John D Isaacs (D) , 1 Gerd R Burmester (D) ${ }^{2}$
}

The past two decades have witnessed the revolution of targeted therapies for immune-mediated inflammatory diseases, largely pioneered for rheumatological indications. These include tumour necrosis factor (TNF) blockade, interleukin (IL)-6 receptor blockade, IL-1 blockade, IL-17 blockade, IL-12/23 blockade, B-cell depletion and costimulation blockade. Other targets, such as type I interferons and granulocyte macrophage-colony stimulating factor (GM-CSF) remain under investigation. ${ }^{1}$ Most recently, janus kinase (JAK) inhibition has provided a small molecule route to targeted interventions. These therapies have been revolutionary, largely because they have provided previously unprecedented efficacy with relative safety. Thus, the risk of a serious infection with a TNF inhibitor is slightly higher than with a conventional synthetic disease-modifying drug such as methotrexate but significantly less than with higher doses of glucocorticoids for example. ${ }^{2} 3$ Similarly, multiple studies have failed to demonstrate an enhanced risk of solid cancers or lymphomas with biological and targeted synthetic disease-modifying anti-rheumatic drugs (DMARDs). ${ }^{4}$ Notably, also treatment with methotrexate which is still the cornerstone of treatment in rheumatoid arthritis (RA), does not have profound immunosuppressive effects, since in the Cardiovascular Inflammation Reduction Trial (CIRT) with non-RA patients the hazard rate for infections was low (1.15 (CI: 1.01 to 1.30)) despite significant comorbidities in the population studied. ${ }^{5}$ These data sit in contrast to immunosuppressive drugs used for indications such as transplantationwhich were also revolutionary in their time. Renal allografts have a 1-year survival of

\footnotetext{
${ }^{1}$ Translational and Clinical Research Institute and Musculoskeletal Unit, Newcastle University and Newcastle upon Tyne Hospitals NHS Foundation Trust, Newcastle upon Tyne, UK

${ }^{2}$ Department of Rheumatology and Clinical Immunology, Charité University Hospital, Berlin, Germany
}

Correspondence to Professor John D Isaacs, Newcastle University Translational and Clinical Research Institute, Faculty of Medical Sciences, Newcastle upon Tyne NE2 4HH, UK; john.isaacs@newcastle.ac.uk
$>90 \%$, using drugs such as tacrolimus, mycophenolate and sometimes glucocorticoids. ${ }^{7}$ However, this success comes at the cost of a significantly heightened risk of serious infections and cancer. ${ }^{89}$ Rheumatologists are cognisant of similar risks when we use glucocorticoids, cyclophosphamide, azathioprine and mycophenolate, particularly in combination.

The way we describe our tools and our actions has a profound effect on the understanding of our patients and other healthcare professionals. The choice between the terms immunosuppressants and immunomodulators to describe the drugs that we use to treat inflammatory rheumatic and musculoskeletal diseases (RMDs) provides a topical and important example. For patients, the difference between their immune system being suppressed, with the implicit susceptibility to infection, and modulation to become more normal, can affect their behaviours in terms of work and social activities. In their COVID-19 guidance, the American College of Rheumatology (ACR) repeatedly distinguishes immunosuppressants (eg, tacrolimus, cyclosporin A (CSA), mycophenolate mofetil (MMF), azathioprine $[\mathrm{AZA}]$ ), biologics and/or JAK inhibitors. ${ }^{10}$ In contrast, the National Institutes of Health (NIH) highlights 'the broad immunosuppressive effect of JAK inhibitors ${ }^{11}$ and the EULAR COVID-19 guidance refers to immunosuppressive treatments, including synthetic DMARDs and biologic DMARDs'. ${ }^{12}$ Thus, there appears to be some uncertainty around how we should describe the drugs that we, as rheumatologists, use daily. Indeed, there was animated discussion among the EULAR COVID-19 Task Force around the most appropriate adjective to use, some members preferring the term 'immunomodulatory', which is also not used in the ACR guidance.

The COVID-19 pandemic has thrust several targeted therapies into the spotlight due to the perception that some serious complications of Severe Acute Respiratory Syndrome-Coronavirus-2 (SARS-CoV-2) infection reflect excess cytokine production locally in the lungs and, potentially, systemically. This has stimulated tremendous opportunities and collaborative research efforts, in new directions and with new partners-rheumatologists working with infectious disease physicians, pulmonologists and intensivists as we join forces to fight COVID-19. Indeed, it is critical that rheumatologists are present at the COVID-19 table, providing insights into the risk:benefit ratio of using cytokine blockade, based on more than two decades of experience. During such discussions, we must not forget normal physiology, for example, that type I interferon is essential for viral defence and GM-CSF for surfactant production by alveolar myeloid cells, stressing the potential importance of timing when using targeted interventions. We should be appropriately cautious when advocating trials of these potent therapies, as we are every day in our clinics, reflecting common sense principles as well as formal guidance.

The important message, when contrasting targeted therapies and immunosuppressants, is that the immune system is a highly sophisticated organ, which adopts a 'belt and braces' approach when protecting our body from foreign invaders. Consequently, when an allograft is detected, multiple immune effector mechanisms are mobilised synchronously, to rapidly eliminate the 'invader'; therefore reversal of allograft rejection requires broadly acting immunosuppressive drugs to disable multiple mechanisms, unavoidably enhancing infection risk in parallel. In contrast, immune-mediated inflammatory diseases (IMIDs) are complex disorders in which multiple, subtle, inherited defects in immune regulatory pathways, combined with environmental triggers, result in the gradual onset of autoreactivity and a chronic inflammatory state. While multiple immuno-inflammatory pathways may be upregulated in tissues, the success of targeted therapies suggest that these operate in series with one or a few dominating. Therefore, targeting TNF in RA, or IL-17 in psoriasis, may restore homeostasis and health, without a major impact on infection risk. Furthermore, unlike allograft rejection, efficacy does not necessitate targeting of underlying autoreactivity.

The immune system is often likened to an army, the different arms of the immune response, and distinct cell subsets, viewed as regiments, with coordinating roles for 'generals' such as dendritic cells, and communicating roles for molecules such as cytokines. Redundancy is a critical virtue of the immune system and of armies-if one element is destroyed, or neutralised, another can often take over. During conflicts of the 20th century, however, relatively untargeted fighting and attacks were used in an attempt to overcome this 
Table 1 Immunosuppressant versus immunomodulatory drugs and their actions. Immunomodulatory drugs are usually biological therapeutics with specific, extracellular effects on a particular pathway/cell type. Immunosuppressive drugs are small molecule therapeutics with selective effects on intracellular pathways. We have placed JAK inhibitors (and methotrexate) as immunomodulatory drugs because, to date, their risk:benefit profile aligns more precisely with this category

\begin{tabular}{|c|c|c|c|}
\hline \multicolumn{2}{|c|}{ Immunosuppressant-broad effects } & \multicolumn{2}{|c|}{ Immunomodulators—specific effects/anti-inflammatory } \\
\hline Drug & Effect & Drug & Effect \\
\hline Azathioprine & Multiple-antiproliferative & TNF inhibition & Specific cytokine blockade \\
\hline Mycophenolate & Purine biosynthesis—antiproliferative, especially lymphocytes & IL-6 receptor blockade & Specific cytokine blockade \\
\hline Cyclophosphamide & Alkylating agent-antiproliferative & Rituximab & B-cell depletion \\
\hline Ciclosporin & Calcineurin inhibitor-inhibition of lymphocyte signalling & Abatacept & Costimulation blockade (T-cell modulator) \\
\hline Tacrolimus & Calcineurin inhibitor-inhibition of lymphocyte signalling & Anakinra & Specific cytokine blockade (IL-1) \\
\hline Glucocorticoids & Multiple genomic effects on immune and inflammatory pathways & IL-17 inhibition & Specific cytokine blockade \\
\hline \multirow[t]{2}{*}{ Sirolimus } & Inhibits mTOR—inhibits lymphocyte signalling and activation & Methotrexate & $\begin{array}{l}\text { Folic acid antagonist-precise mode of action in IMIDs } \\
\text { uncertain }\end{array}$ \\
\hline & & JAK inhibition & Selective inhibition of lymphocyte signalling \\
\hline
\end{tabular}

inherent redundancy, with great loss of life and destruction of infrastructure. The ravages of these conflicts have in part catalysed the modern concept of technologyenabled 'smart warfare', for example, using drones to target critical installations, such as communications systems, incapacitating capabilities while minimising loss of military and civilian life and infrastructure.

Using this analogy, traditional immunosuppression is akin to traditional conflicts-disabling multiple immune elements simultaneously, often using drug combinations. This relatively broad attack on the immune system contrasts with modern targeted therapies, which interrupt communications using cytokine blockade, or disable just a particular type of lymphocyte, or regiment, with rituximab, leaving the remaining immune army unscathed. Of course, targeted therapies still carry risks, particularly if not used with carecombining such treatments leads to more adverse events ${ }^{13}$; and, when used longterm, drugs such as rituximab can eventually weaken immune defences, and reduced IgG levels may presage an increased risk of severe infection. ${ }^{14}$ Similarly, there are circumstances where even a targeted intervention leads to a specific complication due to lack of redundancy-TNF blockade and tuberculosis (TB) reactivation providing a classic example. Perhaps the strongest endorsement of the potency but relative safety of these drugs is our willingness to study them in populations at risk for RA, in an attempt to prevent disease onset. ${ }^{15}$

Having worked with targeted therapies for two decades, rheumatologists now have a unique opportunity to educate colleagues in other specialities about these interventions, which have become our daily bread. Critically, we should take care when describing their effects, to avoid inappropriate assumptions as to their potential complications and adverse effects. Ultimately, the
EULAR Task Force on COVID-19 decided to use the term 'immunosuppressive', 'since it is the fear for and perception of inappropriate suppression of the immune system that leads to the discontinuation of these drugs in the case of COVID-19'. Nonetheless, this approach could reinforce rather than allay concerns, conjuring up the risks of calcineurin inhibitors, cyclophosphamide and glucocorticoids. Equally importantly, immunomodulatory does not equal safety. Used with knowledge, experience, skill, confidence and common sense, however, we can study the potential benefits of modern day, immunomodulatory targeted therapies in the fight against COVID-19, while minimising the potential for serious adverse events. In this way we, as rheumatologists, can help to find effective treatments for the devastating complications of SARS-CoV-2 infection (table 1).

Correction notice This article has been corrected since it published Online First. An author note has been added.

\section{Handling editor Josef S Smolen \\ Twitter John D Isaacs @ProfJohnlsaacs}

Contributors Both authors contributed to the writing of the manuscript.

Funding Work in JDI's laboratory is supported by the National Institute for Health Research Newcastle Biomedical Research Centre based at Newcastle upon Tyne Hospitals NHS Foundation Trust and Newcastle University; and the Research into Inflammatory Arthritis Centre Versus Arthritis. Work in GRB's laboratory is supported by the German Science Foundation (DFG) and the German Ministry of Education and Science.

Disclaimer The views expressed are those of the authors and not necessarily those of the NHS, the NIHR or the Department of Health.

Competing interests JDI: grant from Pfizer, fees for lectures and consulting: AbbVie, Amgen, Eli Lilly, Gilead, Merck \& Co, Roche, UCB. GRB: honoraria for lectures and consulting: AbbVie, Amgen, BMS, Gilead, Janssen, Lilly, Novartis, Pfizer, Sanofi-Aventis, Roche, UCB.

Patient and public involvement Patients and/or the public were not involved in the design, or conduct, or reporting, or dissemination plans of this research.
Patient consent for publication Not required. Provenance and peer review Commissioned; internally peer reviewed.

Author note This Editorial was written before the preliminary results of the dexamethasone arm of the RECOVERY trial were released (1). A total of 2104 patients were randomised to receive dexamethasone $6 \mathrm{mg}$ once per day for ten days and 4321 patients were randomised to usual care alone. Dexamethasone reduced deaths by one-third in ventilated patients (rate ratio 0.65 [95\% confidence interval 0.48 to 0.88 ]; $p=0.0003)$ and by one fifth in other patients receiving oxygen only ( 0.80 [0.67 to 0.96$] ; p=0.0021$ ). There was no benefit among those patients who did not require respiratory support (1.22 [0.86 to 1.75; $p=0.14)$. These data, whilst preliminary, have important implications: They suggest that at least part of the mortality in COVID-19 is driven by hyperinflammation. The timing of therapy is likely to be important - given too early in the illness it could reduce viral clearance. It will be important to examine safety in each patient group, but particularly in those with milder disease. Dexamethasone may become (and in some countries already has become) standard of care for severe COVID-19. This will make it more difficult to demonstrate benefit of other immunomodulators assuming all trial participants receive dexamethasone. Studies will need to be larger, and there will be additional safety considerations. Given the low price of dexamethasone, cost-effectiveness will become an important outcome. Immunomodulator trials should continue, however, to provide insights into the mechanisms driving COVID-19 hyperinflammation, and potentially to provide 'smarter' therapeutic options. References: 1. http://www.ox.ac.uk/news/2020-06-16dexamethasone-reduces-death-hospitalised-patientssevere-respiratory-complications. Accessed 21.6.2020.

This article is made freely available for use in accordance with BMJ's website terms and conditions for the duration of the covid-19 pandemic or until otherwise determined by BMJ. You may use, download and print the article for any lawful, non-commercial purpose (including text and data mining) provided that all copyright notices and trade marks are retained.

(C) Author(s) (or their employer(s)) 2020. No commercial re-use. See rights and permissions. Published by BMJ.

\section{Check for updates}

To cite Isaacs JD, Burmester GR. Ann Rheum Dis 2020;79:991-993.

Received 16 May 2020

Revised 30 May 2020 
Accepted 30 May 2020

Published Online First 11 June 2020

Ann Rheum Dis 2020;79:991-993.

doi:10.1136/annrheumdis-2020-218019

\section{ORCID iDs}

John D Isaacs http://orcid.org/0000-0002-6103-7056

Gerd R Burmester http://orcid.org/0000-0001-75181131

\section{REFERENCES}

1 Baker KF, Isaacs JD. Novel therapies for immunemediated inflammatory diseases: what can we learn from their use in rheumatoid arthritis, spondyloarthritis, systemic lupus erythematosus, psoriasis, Crohn's disease and ulcerative colitis? Ann Rheum Dis 2018:77:175-87.

2 Kang EH, Jin Y, Tong AY, et al. Risk of serious infection among initiators of TNF inhibitors plus methotrexate versus triple therapy for rheumatoid arthritis: a cohort study. Arthritis Care Res 2019. doi:10.1002/acr.24038. [Epub ahead of print: 03 Aug 2019].

3 Sepriano A, Kerschbaumer A, Smolen JS, et al. Safety of synthetic and biological DMARDs: a systematic literature review Informing the 2019 update of the EULAR recommendations for the management of rheumatoid arthritis. Ann Rheum Dis 2020;79:760-70

4 Mercer LK, Regierer AC, Mariette X, et al. Spectrum of lymphomas across different drug treatment groups in rheumatoid arthritis: a European registries collaborative project. Ann Rheum Dis 2017;76:2025-30

5 Ridker PM, Everett BM, Pradhan A, et al. Low-Dose methotrexate for the prevention of atherosclerotic events. N Engl J Med 2019;380:752-62.

6 Solomon DH, Glynn RJ, Karlson EW, et al. Adverse effects of low-dose methotrexate: a randomized trial. Ann Intern Med 2020:172:369-80.

7 Jardine AG, Hartmann A, Holdaas H. Long-Term renal allograft survival: a quiet revolution. Kidney Int 2018:94:853-5.

8 Fishman JA. Infection in organ transplantation. Am J Transplant 2017;17:856-79.

9 Singavi AK, Harrington AM, Fenske TS. Post-Transplant lymphoproliferative disorders.. Cancer Treat Res 2015;165:305-27.

10 Mikuls TR, Johnson SR, Fraenkel L, et al. American College of rheumatology guidance for the management of adult patients with rheumatic disease during the COVID-19 pandemic. Arthritis Rheumatol 2020. doi:10.1002/art.41301. [Epub ahead of print: 29 Apr 2020].
11 National Institutes of Health (NIH). Coronavirus disease 2019 (COVID-19) treatment guidelines, 2020. Available: https://files.covid19treatmentguidelines nih.gov/guidelines/covid19treatmentguidelines. pdf.[Accessed 10th June 2020].

12 Landewé RBM, Machado PM, Kroon F, et al. EULAR provisional recommendations for the management of rheumatic and musculoskeletal diseases in the context of SARS-CoV-2. Ann Rheum Dis 2020;79:851-8.

13 Genovese MC, Cohen S, Moreland L, et al. Combination therapy with etanercept and anakinra in the treatment of patients with rheumatoid arthritis who have been treated unsuccessfully with methotrexate. Arthritis Rheum 2004:50:1412-9.

14 van Vollenhoven RF, Emery P, Bingham CO, et al. LongTerm safety of rituximab in rheumatoid arthritis: 9.5year follow-up of the global clinical trial programme with a focus on adverse events of interest in RA patients. Ann Rheum Dis 2013;72:1496-502.

15 Al-Laith M, Jasenecova M, Abraham S, et al. Arthritis prevention in the pre-clinical phase of RA with abatacept (the APIPPRA study): a multi-centre, randomised, double-blind, parallel-group, placebocontrolled clinical trial protocol. Trials 2019;20:429. 